\title{
Granulocyte-colony stimulating factor administration for neurological improvement in patients with postrehabilitation chronic incomplete traumatic spinal cord injuries: a double-blind randomized controlled clinical trial
}

\author{
Nazi Derakhshanrad, MD, PhD, ${ }^{1}$ Hooshang Saberi, MD, MPH, ${ }^{1,2}$ Mir Saeed Yekaninejad, PhD, ${ }^{3}$ \\ Mohammad Taghi Joghataei, PhD, ${ }^{4,5}$ and Abdolreza Sheikhrezaei, MD²
}

\begin{abstract}
${ }^{1}$ Brain and Spinal Cord Injury Research Center (BASIR), Neuroscience Institute, Imam Khomeini Hospital, Tehran University of Medical Sciences; ' $D$ Department of Neurosurgery, Imam Khomeini Hospital, Tehran University of Medical Sciences; ${ }^{3}$ Department of Epidemiology and Biostatistics, School of Public Health, Tehran University of Medical Sciences; and ${ }^{4}$ Cellular and Molecular Research Center and ${ }^{5}$ Neuroscience Department, School of Advanced Technologies in Medicine, Iran University of Medical Sciences, Tehran, Iran
\end{abstract}

OBJECTIVE Granulocyte-colony stimulating factor (G-CSF) is a major growth factor for activation and differentiation of granulocyte colonies in the bone marrow. This cytokine has been widely and safely employed in different conditions over many years. The purpose of this study was to investigate the efficacy of G-CSF administration for traumatic spinal cord injury (TSCI).

METHODS This double-blind parallel randomized, placebo-controlled, clinical trial, a phase III study, was performed from June 2013 to June 2016 in the Brain and Spinal Cord Injury Research (BASIR) center at Tehran University of Medical Sciences (TUMS). It included 120 patients with incomplete chronic TSCI, American Spinal Injury Association (ASIA) Impairment Scale (AIS) B, C, or D, of at least 6 months' duration. Sixty patients were allocated into the treatment group and 60 patients into the control group. All the patients had completed an outpatient rehabilitation program in the postacute period and were in a neurological and functional plateau. Patients were assessed with the ASIA grading system, the Spinal Cord Independence Measure (SCIM-III), and the International Association of Neurorestoratology-Spinal Cord Injury Functional Rating Scale (IANR-SCIFRS) just before intervention and at 1, 3, and 6 months after 7 subcutaneous administrations of $300 \mu \mathrm{g} / \mathrm{day}$ of G-CSF in the treatment group and placebo in the control group (administered once per day over the course of 1 week). Randomization was performed with randomized block design, and the patients and evaluators were blinded regarding the treatment groups. One patient did not receive the entire allocated intervention and 5 patients were lost to follow-up. Thus data from 114 patients were included in the analysis.

RESULTS One hundred twenty patients were randomized and allocated into the study groups. Among them, 56 patients $(93.3 \%)$ in the G-CSF group and 58 patients $(96.6 \%)$ in the placebo group completed the study protocol. After 6 months of follow-up, AIS in the placebo group remained unchanged, whereas in the G-CSF group, 1 patient improved from AIS $B$ to $C$, and 4 patients improved from AIS $C$ to $D$. The mean ( $\pm S E$ ) improvement in ASIA motor score in the G-CSF group was $5.5 \pm 0.62$, which was significantly more than in the placebo group $(0.77 \pm 0.20)(p<0.001)$. The mean light touch and pinprick sensory scores, respectively, increased by $6.1 \pm 1.1$ and $8.7 \pm 1.5$ in the G-CSF group and by 1.3 \pm 0.52 and $0.89 \pm 0.44$ scores in the placebo group $(p<0.001)$. Evaluation of functional improvement by the IANRSCIFRS instrument revealed significantly more improvement in the G-CSF group $(3.5 \pm 0.37)$ than in the placebo group

\footnotetext{
ABBREVIATIONS AIS = ASIA Impairment Scale; ASIA = American Spinal Injury Association; BASIR = Brain and Spinal Cord Injuries Research; CBC = complete blood count; CONSORT = Consolidated Standards of Reporting Trials; G-CSF = granulocyte-colony stimulating factor; HO = heterotopic ossification; IANR-SCIFRS = International Association of Neurorestoratology-Spinal Cord Injury Functional Rating Scale; LUQ = left upper quadrant; MAS = Modified Ashworth Scale; MI = myocardial infarction; SCl = spinal cord injury; SCIM-III = Spinal Cord Independence Measure version III; SCIPUS = SCI Pressure Ulcer Scale; SD = standard deviation; SE = standard error; TSCI = traumatic spinal cord injury; TUMS = Tehran University of Medical Sciences; VAS = visual analog scale; WBC = white blood cell.
}

SUBMITTED July 8, 2017. ACCEPTED November 1, 2017.

INCLUDE WHEN CITING Published online April 27, 2018; DOI: 10.3171/2017.11.SPINE17769. 
$(0.41 \pm 0.12)(p<0.001)$. Also, a significant difference was observed in functional improvement between the 2 groups as measured by SCIM-III instrument ( $7.5 \pm 0.95$ vs $2.1 \pm 0.51, p<0.001)$.

CONCLUSIONS Administration of G-CSF for incomplete chronic spinal cord injuries is associated with significant motor, sensory, and functional improvement.

Clinical trial registration no.: IRCT201108297441N1 (www.irct.ir)

https://thejns.org/doi/abs/10.3171/2017.11.SPINE17769

KEYWORDS spinal cord injury; granulocyte-colony stimulating factor; neurological improvement

$\mathrm{T}$ RAUMATIC spinal cord injury (TSCI) is a devastating neurological consequence of trauma causing longterm morbidity and marked changes in patients' lifestyles. ${ }^{11}$ There are many rehabilitation measures for management of spinal cord injury (SCI) after early decompression, fixation, and clinical stabilization, but studies have shown that residual neurological and functional deficits remain after completion of the standard rehabilitation programs. ${ }^{1,2,10}$ These neurological deficits are associated with high annual costs for treatment of complications and direct and indirect costs for nursing, equipment, and social supports. Therefore, not only the patients but also the health care providers have a strong desire for even a small improvement in patients' neurological status.

After primary SCI and secondary inflammatory responses, ${ }^{29}$ incomplete neuroregenerative responses in the spinal cord make it a potential target for local or systemic regenerative treatments. ${ }^{21}$

Various neurorestorative agents have been proposed for treatment of acute and chronic SCI, including steroids, the ganglioside GM-1, Cethrin, riluzole, nimodipine, minocycline, ${ }^{20}$ erythropoietin, ${ }^{5}$ and granulocyte-colony stimulating factor (G-CSF), with various application schedules and outcomes. . $^{8,26,34,35,39}$ The use of G-CSF has been suggested to be a viable alternative to bone marrow stem cell autografts in experimental models of SCI. ${ }^{16}$ Therefore GCSF may be a candidate drug for SCI management that can be applied for the clinical evaluation without ethical or methodological problems, ${ }^{12}$ given that G-CSF is among the few growth factors that have been shown to be safe for clinical applications. ${ }^{9}$

On the other hand, the rapidly growing knowledge regarding molecular and cellular treatments for experimental models of SCI has led to phase I trials with local ethics board approvals in some centers..$^{9,34,39}$ Nevertheless widespread application of new treatments requires welldesigned randomized trials. Phase III studies require a blinded control group receiving placebo, and therefore noninvasive treatments are better suited in this model.

On the basis of the available experimental and clinical results described earlier, we have previously conducted phase $\mathrm{I}^{9}$ and phase $\mathrm{I} / \mathrm{IIa}^{32}$ clinical trials to confirm the safety and feasibility of G-CSF administration for patients with chronic TSCI. In this trial, a phase III study, we are trying to compare the efficacy of subcutaneous G-CSF with placebo in chronic incomplete TSCI, reporting on neurological as well as functional findings in the 2 groups.

\section{Methods Study Design}

This double-blind parallel randomized, placebo-controlled, clinical trial (phase III study) was conducted from June 2013 to June 2016 in the Brain and Spinal Cord Injuries Research (BASIR) center at Tehran University of Medical Sciences (TUMS). The study was approved by the local institutional review board/ethics committee of TUMS. The study was registered in the Iranian Registry of Clinical Trials (IRCT; registration no. IRCT201108297441N1, www.irct.ir) before enrollment of the participants. The study adheres to the criteria of the revised Consolidated Standards of Reporting Trials (CONSORT) 2010 statement for reporting randomized trials, and ethical codes conformed to the Declaration of Helsinki.

The patients were selected from outpatient postrehabilitation SCI cases registered in BASIR center. One hundred twenty postrehabilitation patients with chronic incomplete TSCI were chosen and enrolled after providing written informed consent for the study.

Baseline evaluations included duration of injury, neurological level, severity of injury by ASIA Impairment Scale (AIS grade range $\mathrm{A}-\mathrm{E}$ ), and current complications. Complications were evaluated as follows: neuropathic pain by visual analog scale (VAS; range 0-100), spasticity by Modified Ashworth Scale (MAS; score range 1-4), pressure ulcers (PUs) by SCI Pressure Ulcer Scale (SCIPUS; grade I-IV). ${ }^{36}$ Neurological changes were assessed by the American Spinal Injury Association (ASIA) scale (motor score range 0-100, light touch score range 0-112, pinprick score range 0-112). ${ }^{25}$ The Spinal Cord Independence Measure version III (SCIM-III) (score range 0-100)22 as well as the International Association of Neurorestoratology-Spinal Cord Injury Functional Rating Scale (IANR-SCIFRS) (score range $0-48)^{19}$ were used to assess functional changes.

\section{Study Participants}

One hundred fourteen TSCI patients were recruited for the study in the BASIR center at Imam Hospital, TUMS. Patients aged between 18-60 years were enrolled in the study. Patients with incomplete (AIS = B, C, D) TSCIs, having accomplished at least 3 months of active rehabilitation according to the consortium of spinal cord medicine guidelines, and at least 6 months duration of SCI, were selected. They all had undergone decompression and fixation if necessary in the acute setting. Neurological examination was performed and patients with upper motor neu- 
ron type injury without lower neuron involvement were selected. All of the patients declared availability for the follow-up meetings.

Patients were excluded if they had 1) AIS A (complete cord transection); 2) joint motion restriction (beginning of heterotopic ossification (HO) and/or joint contractures); 3) a major complication such as grade 3 or 4 pressure sores, urinary tract infection with sepsis, and pneumonia, deep vein thrombosis (DVT), implanted instrument failure, etc.; 4) severe atrophy of the spinal cord on MRI $(<10 \%$ of normal adjacent spinal cord diameter) and/or concomitant syringomyelia; 5) major associated injury such as head trauma and/or brain injury, or fracture of upper or lower limbs leading to deformity and ankylosis; 6) abnormal findings on baseline complete blood count (CBC); 7) history of blood dyscrasia, and/or hematological abnormality (sickle cell disorder, platelet abnormality); 8) history of cardiovascular disease such as myocardial infarction (MI) and/or arrhythmia; 9) active peptic ulcer disease; 10) pregnancy or breast feeding; 11) history of severe hypersensitivity to Escherichia coli products and protein derivatives, drugs of this origin, or drugs that increase neutrophil count (such as lithium); 12) any systemic disease contraindicating G-CSF administration; or 13) adverse reactions to the G-CSF. In addition patients were excluded in the event of noncompliance or request to leave the study.
Among the 532 patients referred to outpatient clinic of BASIR center, 412 patients were excluded in accordance with the flow diagram shown in Fig. 1. Three hundred ninety-eight patients did not meet the inclusion criteria-354 because of AIS A SCI, 34 due to age $>60$ years or age $<18$ years, 3 because of complicated grade 3 or 4 pressure sores, 2 because of DVT, 1 because of metallic implant failure, 1 because of $\mathrm{HO}, 1$ because of concomitant syringomyelia, 1 because of a history of MI, and another due to platelet abnormality. Eight patients declined to participate, and 6 patients were excluded for other reasons such as traveling difficulty to the center. Finally 120 patients with TSCI, were randomized and allocated into the study groups (60 to the treatment group and 60 to the control group). All the patients had completed an outpatient rehabilitation program in the post-acute period and were in neurological and functional plateau.

\section{Study Protocol}

At the screening visit, after completion of the demographic and clinical assessments, traumatic etiology of the lesion was confirmed. Severity of SCI was evaluated based on AIS grading at the next step. After informed consent was obtained, a baseline CBC was taken and patients' neurological condition was assessed with the ASIA sensorymotor scale. ${ }^{26}$ Functional assessment was performed with

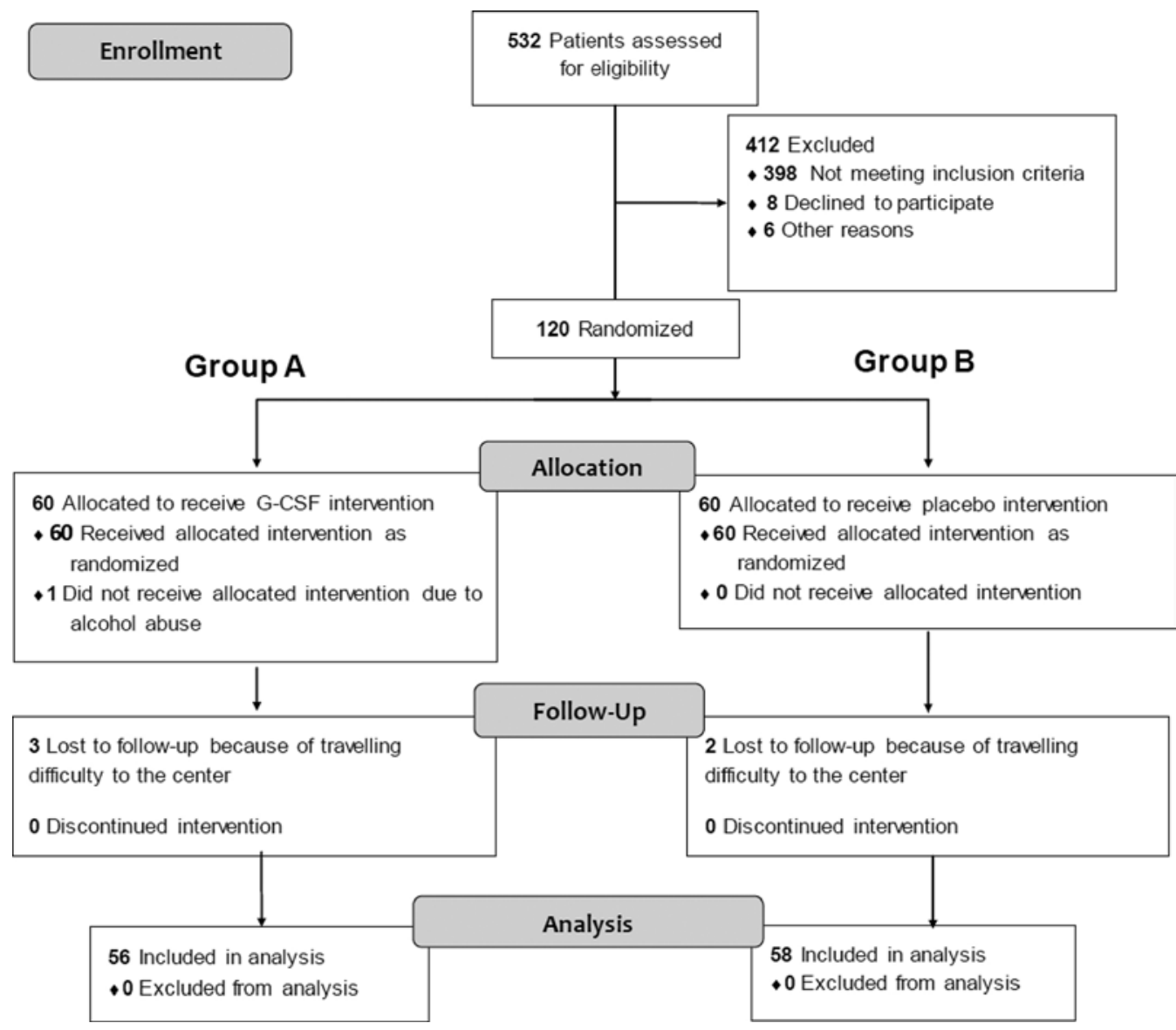

FIG. 1. Flow diagram for trial. Prepared using the CONSORT 2010 Flow Diagram template (available at http://www.consortstatement.org/). 
SCIM-III ${ }^{22}$ and IANR-SCIFRS. ${ }^{19}$ Randomization was performed with a randomized block design, and allocation was performed with the sealed-envelope method. Enrollment of the participants was performed by the attending neurosurgeon (H.S.), blinded to the treatment groups. Random allocation was performed based on a computergenerated list obtained from an investigator with no clinical involvement in the trial. Then by the sealed-envelope method, an independent staff nurse assigned the participants to interventions. Patients and evaluators were blinded to treatment group assignments-G-CSF and placebo vials had the same cover shape but different codes. The vials for patients in the treatment group contained $300 \mu \mathrm{g}$ of G-CSF (1-ml filgrastim, Neupogen, Amgen); patients were administered the contents of 1 vial a day subcutaneously for 7 consecutive days. The vials for the control group contained $1 \mathrm{ml}$ of normal saline $0.9 \%$, and same administration regimen was followed. A CBC was performed with a Sysmex XT-1800i hematology analyzer daily for the next 7 days. The test was performed again at 3 and 6 months after treatment to assess chronic hematological effects. At the 1-, 3-, and 6-month follow-up visits, the patients were assessed for functional and neurological status. A single examiner performed the neurological assessments and another examiner performed the functional assessments in all cases from the baseline examination through the last follow-up visit. Therefore our statistician did not recommend interrater reliability analysis.

\section{Primary and Secondary Outcome Measures}

The primary outcome measure was the efficacy of GCSF administration for chronic incomplete TSCI. Change in ASIA $^{25}$ motor or sensory scores was considered the primary outcome measure. Functional changes, evaluated by SCIM-III ${ }^{22}$ and IANR-SCIFRS, ${ }^{19}$ were considered as secondary outcome measure. Evaluations were measured at baseline, and at the 1-month, 3-month, and 6-month intervals postintervention. Safety was also evaluated as an important outcome measure. Any evidence of complications up to 1 year after intervention was recorded regularly and reported.

Criteria for stopping treatment included a white blood cell (WBC) count $>50,000 / \mathrm{mm}^{3}$ in the CBC. Also, any sign of hypersensitivity reaction and/or thrombocytopenia was an indication for discontinuation of the injections.

One hundred and twenty cases were randomized and allocated to the 2 groups (60 patients per group). In the treatment group, 1 patient did not receive allocated intervention due to alcohol overdose after the third injection and 3 patients were lost to follow-up due to transport problems. In the control group, 2 patients were lost to follow-up due to transport problems. None of these patients reported any complication. A total of 114 patients completed the follow-up period. There were no missing data for any of these cases. The analysis included 56 patients in G-CSF group and 58 patients in placebo group.

\section{Statistical Analysis}

Descriptive results are presented as mean and standard deviation (SD) for continuous variables and frequency (\%) for categorical variables. Statistical comparisons of qualitative variables between 2 groups were made by means of the chi-square or Fisher exact test. Changes in quantitative variables in G-CSF and placebo groups were compared with independent sample t-tests.

The mean and standard error (SE) were calculated for outcome measures including ASIA motor, light touch, pinprick, SCIM III, and IANR-SCIFRS scores and the changes were reported. Analysis of covariance was used for comparing the change in outcome measure in the 2 groups, with treatment group as fixed effect and baseline score as a covariate. A p value $<0.05$ was considered statistically significant. Data analysis was performed using SPSS software version 22 (IBM Corp.). Sample size was calculated for comparing 2 independent groups with medium effect size (Cohen's $d=0.5), 0.85$ statistical power, and 0.05 probability of Type I error.

\section{Results}

\section{Patient Characteristics}

A total of 120 patients (mean age 36.3 years [SD 11.1 years], 105 [87.5\%] male) were randomized into this single-center study (60 to the G-CSF group and 60 to the placebo group) from June 2013 for 2 years. The follow-up period was 1 year after recruitment for each patient. Of the 120 patients enrolled, 56 patients (93.3\%) in the GCSF group and 58 patients (96.6\%) in the placebo group finished the study (Fig. 1).

The mean age of the patients in G-CSF group was 35.1 years (SD 11.2 years), and the mean age in the placebo group was 37.2 years (SD 10.6 years). The percentage of male patients was $87.5 \%$ in the G-CSF group and $82.7 \%$ in the placebo group. The neurological level was cervical in 28 , thoracic in 21 , and lumbar (L-1) in 7 patients in the GCSF group versus cervical in 30, thoracic in 20, and lumbar (L-1) in 8 patients in the placebo group $(\mathrm{p}=0.939)$. The distribution of AIS grades was $14 \mathrm{~B}, 29 \mathrm{C}$, and $13 \mathrm{D}$ in the GCSF group versus 20 AIS B, 27 AIS C, and 11 AIS D in the placebo group $(p=0.532)$. The mean duration of SCI (time from injury to study entry) was 53.7 months in the G-CSF group versus 51 months in the placebo group $(p=0.799)$.

There were no significant differences in demographic characteristics regarding age, sex, or duration or cause of SCI between the study groups. Neurological level, AIS grade, rostrocaudal length of the lesion on MRI, and baseline WBC count did not show significant between-groups differences (Table 1). Comparison for AIS grades did not show significant difference between cervical $(p=0.819)$ and thoracolumbar cases $(\mathrm{p}=0.598)$.

\section{Neurological Assessment}

The mean improvement in ASIA motor score in the G-CSF group was 5.5 (SE 0.62), which was significantly greater than in the placebo group (0.77 [SE 0.20]) (p < 0.001). In comparison to the placebo group, the G-CSF group also showed significantly greater improvement in mean light touch scores (6.1 [SE 1.1] vs 1.3 [SE 0.52], p < $0.001)$ and pinprick sensory scores (8.7 [SE 1.5] vs 0.89 [SE 0.44], p < 0.001) (Table 2).

Regarding AIS grade, $7.1 \%$ of AIS B patients changed 
TABLE 1. Demographic and clinical characteristics of patients in the G-CSF and placebo groups

\begin{tabular}{|c|c|c|c|}
\hline Characteristics & G-CSF Group $(n=56)$ & Placebo Group $(n=58)$ & p Value \\
\hline Age in yrs at time of injection, mean & 35.1 (SD 11.2) & 37.2 (SD 10.6) & 0.308 \\
\hline Male sex & $49(87.5 \%)$ & $52(82.7 \%)$ & 0.717 \\
\hline Length of time since $\mathrm{SCl}$ in mos, mean & 53.7 (SD 0.06) & 51 (SD 0.04) & 0.799 \\
\hline Education in yrs, mean & 10.1 (SD 4.2) & 9.4 (SD 3.8) & 0.304 \\
\hline Marital status & & & 0.369 \\
\hline Single & $24(42.9 \%)$ & $20(34.5 \%)$ & \\
\hline Married & $29(51.8 \%)$ & $31(53.4 \%)$ & \\
\hline Divorced/separated/widowed & $3(5.4 \%)$ & $7(12.1 \%)$ & \\
\hline Occupational status & & & 0.262 \\
\hline Employed & $14(25 \%)$ & $11(19 \%)$ & \\
\hline Housekeeper (females) & $2(3.6 \%)$ & $3(5.2 \%)$ & \\
\hline Left job (temporary)/fired & $27(48.2 \%)$ & $24(41.4 \%)$ & \\
\hline Retired & $5(8.9 \%)$ & $14(24.1 \%)$ & \\
\hline Remained unemployed & $8(14.3 \%)$ & $6(10.3 \%)$ & \\
\hline Etiology of SCl & & & 0.694 \\
\hline MVA & $41(73.2 \%)$ & $35(60.3 \%)$ & \\
\hline Fall & $7(12.5 \%)$ & $12(20.7 \%)$ & \\
\hline Sport & $2(3.6 \%)$ & $3(5.2 \%)$ & \\
\hline Violence & $2(3.6 \%)$ & $3(5.2 \%)$ & \\
\hline Heavy drop & $4(7.1 \%)$ & $5(8.6 \%)$ & \\
\hline Neurological level of injury & & & 0.939 \\
\hline Cervical & $28(50 \%)$ & $30(51.7 \%)$ & \\
\hline Thoracic & $21(37.5 \%)$ & $20(34.5 \%)$ & \\
\hline Lumbar & $7(12.5 \%)$ & $8(13.8 \%)$ & \\
\hline AIS grade & & & 0.532 \\
\hline $\mathrm{B}$ & $14(25.0 \%)$ & $20(34.5 \%)$ & \\
\hline C & $29(51.8 \%)$ & $27(46.6 \%)$ & \\
\hline $\mathrm{D}$ & $13(23.2 \%)$ & $11(19.0 \%)$ & \\
\hline Length of lesion in $\mathrm{mm}$, mean & 17.76 (SD 13.4) & 17.76 (SD 14.8) & 1.000 \\
\hline \multicolumn{4}{|l|}{ WBC count, mean } \\
\hline Before treatment & 7,000 (SD 219) & 6,877 (SD 1,507) & 0.678 \\
\hline After treatment & 29,240 (SD 7,750) & 7,451 (SD 2,880) & $<0.001$ \\
\hline
\end{tabular}

MVA = motor vehicle accident.

to AIS C, and $92.9 \%$ remained unchanged. Of the AIS C patients, $13.8 \%$ changed to AIS D, and $86.2 \%$ were unchanged. None of the AIS grade D patients had a change in AIS grade (Fig. 2). There was no AIS change in the placebo group.

\section{Functional Assessment}

Evaluation of functional outcomes by the IANRSCIFRS instrument revealed significantly greater changes in the G-CSF group (mean 3.5 [SE 0.37]) than in the placebo group (mean 0.41 [SE 0.12], $\mathrm{p}<0.001$ ). A significant between-groups difference in functional improvement was observed with the SCIM-III instrument (7.5 [SE 0.95] vs 2.1 [SE 0.51], $\mathrm{p}<0.001$ ) (Table 2).

Regarding SCIM-III subscale changes, the G-CSF group had significantly greater changes in feeding $(\mathrm{p}=$ $0.014)$, bathing upper body ( $\mathrm{p}=0.003)$, dressing upper body $(\mathrm{p}=0.014)$, dressing lower body and grooming $(\mathrm{p}$
$=0.02)$, bladder management $(\mathrm{p}=0.009)$, bowel management $(\mathrm{p}=0.002)$, mobility in bed $(\mathrm{p}=0.04)$, mobility outdoors $(p=0.004)$, and stair management $(p=0.01)$ than the placebo group (Fig. 3).

Regarding IANR-SCIFRS subscale changes, the GCSF group had significant greater improvement in the eating and drinking $(p<0.001)$, grooming $(p=0.004)$, writing $(\mathrm{p}=0.015)$, standing $(\mathrm{p}=0.001)$, walking without brace $(\mathrm{p}=0.003)$, sitting $(\mathrm{p}<0.001)$, turning body over $(\mathrm{p}=0.01)$, transfers: bed to chair/wheelchair $(\mathrm{p}=0.01)$, dressing $(\mathrm{p}=0.02)$, bladder and bowel control subscales ( $\mathrm{p}$ $<0.001)$, muscular tension ( $\mathrm{p}=0.003)$, and sexual function $(\mathrm{p}<0.001)$ than the placebo group (Fig. 4).

\section{Subgroup Analysis}

A subgroup analysis was performed after subdividing the groups by neurological level (cervical or thoracolumbar region). Comparison of the cervical versus thoraco- 
TABLE 2. Comparison of the neurological (based on ASIA) and functional (based on SCIM-III and FRS) scores in the G-CSF and placebo groups before injection and at follow-up

\begin{tabular}{|c|c|c|c|}
\hline Variable & $\begin{array}{c}\text { G-CSF } \\
\text { Group } \\
(n=56)\end{array}$ & $\begin{array}{c}\text { Placebo } \\
\text { Group } \\
(n=58)\end{array}$ & $\begin{array}{c}p \\
\text { Value }\end{array}$ \\
\hline \multicolumn{4}{|c|}{ Motor score (range 0-100) } \\
\hline Before injection & $55.7 \pm 3.0$ & $55.3 \pm 2.8$ & 0.93 \\
\hline 1 mo follow-up & $58.6 \pm 3.0$ & $55.6 \pm 2.8$ & $<0.001$ \\
\hline 3 mos follow-up & $60.0 \pm 3.1$ & $56.0 \pm 2.8$ & $<0.001$ \\
\hline 6 mos follow-up & $61.2 \pm 3.1$ & $56.1 \pm 2.8$ & $<0.001$ \\
\hline Change & $5.5 \pm 0.62$ & $0.77 \pm 0.20$ & $<0.001$ \\
\hline \multicolumn{4}{|c|}{ Light touch score (range 0-112) } \\
\hline Before injection & $87.6 \pm 3.3$ & $90.4 \pm 3.1$ & 0.53 \\
\hline 1 mo follow-up & $90.4 \pm 3.0$ & $90.9 \pm 3.1$ & $<0.001$ \\
\hline 3 mos follow-up & $92.3 \pm 3.0$ & $91.3 \pm 3.1$ & $<0.001$ \\
\hline 6 mos follow-up & $93.7 \pm 3.0$ & $91.8 \pm 3.0$ & $<0.001$ \\
\hline Change & $6.1 \pm 1.1$ & $1.3 \pm 0.52$ & $<0.001$ \\
\hline \multicolumn{4}{|c|}{ Pinprick score (range 0-112) } \\
\hline Before injection & $74.5 \pm 3.8$ & $75.5 \pm 3.9$ & 0.84 \\
\hline 1 mo follow-up & $78.4 \pm 3.6$ & $75.9 \pm 3.9$ & $<0.001$ \\
\hline 3 mos follow-up & $80.4 \pm 3.6$ & $76.3 \pm 3.9$ & $<0.001$ \\
\hline 6 mos follow-up & $83.2 \pm 3.6$ & $76.4 \pm 3.9$ & $<0.001$ \\
\hline Change & $8.7 \pm 1.5$ & $0.89 \pm 0.44$ & $<0.001$ \\
\hline \multicolumn{4}{|c|}{ SCIM III score (range 0-100) } \\
\hline Before injection & $52.0 \pm 3.1$ & $52.9 \pm 3.1$ & 0.85 \\
\hline 1 mo follow-up & $55.1 \pm 3.1$ & $53.3 \pm 3.1$ & $<0.001$ \\
\hline 2 mos follow-up & $57.6 \pm 3.1$ & $54.6 \pm 3.1$ & $<0.001$ \\
\hline 6 mos follow-up & $59.6 \pm 3.1$ & $55.0 \pm 3.1$ & $<0.001$ \\
\hline Change & $7.5 \pm 0.95$ & $2.1 \pm 0.51$ & $<0.001$ \\
\hline \multicolumn{4}{|c|}{ Self Care (range 0-20) } \\
\hline Before injection & $12.0 \pm 0.9$ & $12.6 \pm 0.9$ & 0.64 \\
\hline 1 mo follow-up & $12.4 \pm 0.9$ & $12.6 \pm 0.9$ & $<0.001$ \\
\hline 3 mos follow-up & $12.9 \pm 0.8$ & $12.7 \pm 0.9$ & $<0.001$ \\
\hline 6 mos follow-up & $13.1 \pm 0.8$ & $12.8 \pm 0.9$ & $<0.001$ \\
\hline Change & $1.1 \pm 0.24$ & $0.20 \pm 0.06$ & $<0.001$ \\
\hline \multicolumn{4}{|l|}{$R S M$ (range 0-40) } \\
\hline Before injection & $23.4 \pm 1.1$ & $23.6 \pm 1.0$ & 0.93 \\
\hline 1 mo follow-up & $25.2 \pm 1.0$ & $23.8 \pm 1.0$ & $<0.001$ \\
\hline 3 mos follow-up & $26.6 \pm 1.1$ & $24.7 \pm 1.0$ & 0.001 \\
\hline 6 mos follow-up & $27.5 \pm 1.1$ & $24.9 \pm 1.0$ & $<0.001$ \\
\hline Change & $4.0 \pm 0.60$ & $1.3 \pm 0.41$ & $<0.001$ \\
\hline \multicolumn{4}{|c|}{ Mobility: RT (range 0-10) } \\
\hline Before injection & $7.2 \pm 0.4$ & $7.1 \pm 0.4$ & 0.84 \\
\hline 1 mo follow-up & $7.5 \pm 0.4$ & $7.2 \pm 0.4$ & 0.033 \\
\hline 3mos follow-up & $7.7 \pm 0.4$ & $7.2 \pm 0.4$ & 0.025 \\
\hline 6 mos follow-up & $7.9 \pm 0.4$ & $7.3 \pm 0.4$ & 0.007 \\
\hline Change & $0.67 \pm 0.18$ & $0.17 \pm 0.08$ & 0.012 \\
\hline \multicolumn{4}{|c|}{ Mobility: IO (range 0-30) } \\
\hline Before injection & $9.5 \pm 1.1$ & $9.6 \pm 1.1$ & 0.94 \\
\hline 1 mo follow-up & $10.0 \pm 1.1$ & $9.5 \pm 1.1$ & 0.008 \\
\hline
\end{tabular}

CONTINUED IN NEXT COLUMN »
» CONTINUED FROM PREVIOUS COLUMN

TABLE 2. Comparison of the neurological (based on ASIA) and functional (based on SCIM-III and FRS) scores in the G-CSF and placebo groups before injection and at follow-up

\begin{tabular}{cccc}
\hline Variable & $\begin{array}{c}\text { G-CSF } \\
\text { Group } \\
(n=56)\end{array}$ & $\begin{array}{c}\text { Placebo } \\
\text { Group } \\
(n=58)\end{array}$ & $\begin{array}{c}p \\
\text { Value }\end{array}$ \\
\hline $\begin{array}{c}\text { SCIM III score (range 0-100) } \\
\text { (continued) }\end{array}$ & & & \\
\hline $\begin{array}{c}\text { Mobility: IO (range 0-30) } \\
\text { (continued) }\end{array}$ & & & \\
\hline 3 mos follow-up & $10.5 \pm 1.1$ & $9.7 \pm 1.1$ & 0.003 \\
\hline 6 mos follow-up & $11.1 \pm 1.1$ & $9.8 \pm 1.1$ & 0.003 \\
\hline Change & $1.6 \pm 0.41$ & $0.20 \pm 0.21$ & 0.003 \\
\hline Before injection & & & \\
\hline 1 mo follow-up & $29.2 \pm 1.5$ & $28.8 \pm 1.4$ & 0.85 \\
\hline 3 mos follow-up & $30.5 \pm 1.5$ & $28.8 \pm 1.4$ & $<0.001$ \\
\hline 6 mos follow-up & $31.7 \pm 1.5$ & $29.0 \pm 1.4$ & $<0.001$ \\
\hline Change & $32.7 \pm 1.5$ & $29.2 \pm 1.4$ & $<0.001$ \\
\hline IANR-SCIFRS score (range 0-48) & $3.5 \pm 0.37$ & $0.41 \pm 0.12$ & $<0.001$ \\
\hline
\end{tabular}

10 = indoors and outdoors, on even surface; RSM = Respiration and Sphincter Management; RT = room toilet.

Data are mean $\pm \mathrm{SE}$

lumbar subgroups did not show any significant differences within either the G-CSF group or the placebo group, whereas between-groups comparisons (i.e., G-CSF vs placebo) were significant in both the cervical and thoracolumbar subgroups, as shown in Table 3.

\section{White Blood Cell Count}

A CBC was performed before any of the study injections were administered, as well as daily after each injection, for 7 days. The mean postinjection WBC count in the G-CSF group was 29,240 cells $/ \mathrm{mm}^{3}$ (SD 7,750 cells $/ \mathrm{mm}^{3}$ ) and 7,451 $( \pm 2,880)$ cells $/ \mathrm{mm}^{3}$ in the placebo group, showing a significant increase in G-CSF group compared with the placebo group $(\mathrm{p}<0.001)$.

\section{Systemic Side Effects}

In the G-CSF group, there were $5(8.9 \%)$ patients with bone pain, $1(1.8 \%)$ with muscle pain, $1(1.8 \%)$ with headache, $2(3.5 \%)$ with nausea and vomiting, 1 (1.8\%) with fever, $1(1.8 \%)$ case of transient itching and skin rash, and $1(1.8 \%)$ patient with left upper quadrant (LUQ) pain without splenomegaly on sonography. All complications were transient and subsided spontaneously within 1 week after the last dose of G-CSF. In the placebo group, 1 patient experienced transient headache, nausea, vomiting, and LUQ pain during injections.

\section{Neuropathic Pain and Spasticity}

Seven patients $(12.5 \%)$ in the G-CSF group had increased neuropathic pain ( 3 patients had a 20 -point increase in pain severity, based on VAS, and 4 patients had an increase in the frequency of pain attacks). This effect lasted up to 1 year and was amenable to GABA-agonist 


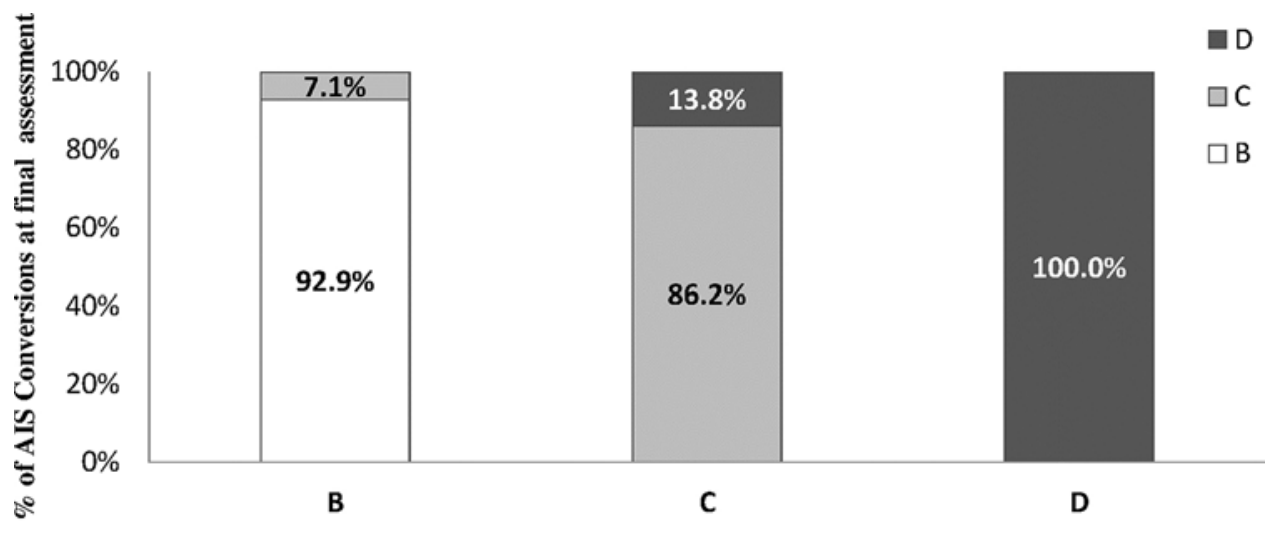

\section{AIS at baseline}

FIG. 2. Percentage of AIS conversions in G-CSF group at final assessment expressed based on pretreatment AIS.

dose adjustment. Another patient (1.8\%) showed increased frequency of spasticity episodes, which were controlled by conservative management. On the other hand, 2 patients $(3.5 \%)$ had decreased neuropathic pain (range of decrease 20-30 points, based on VAS) from 1 to 3 months after injections. Five patients $(8.9 \%)$ showed decreased spasticity. Four patients showed a decrease by 1 score level based on MAS scoring and 1 patient had a reduced number of spasticity episodes in the 1st year of follow-up.

In the placebo group, 5 patients $(8.6 \%)$ reported increased neuropathic pain (2 with 20-30 point increases in VAS scores, 3 with increased frequency) 1-3 months after injection that was amenable to transient GABA-agonist therapy. There were 4 individuals $(6.9 \%)$ with increased

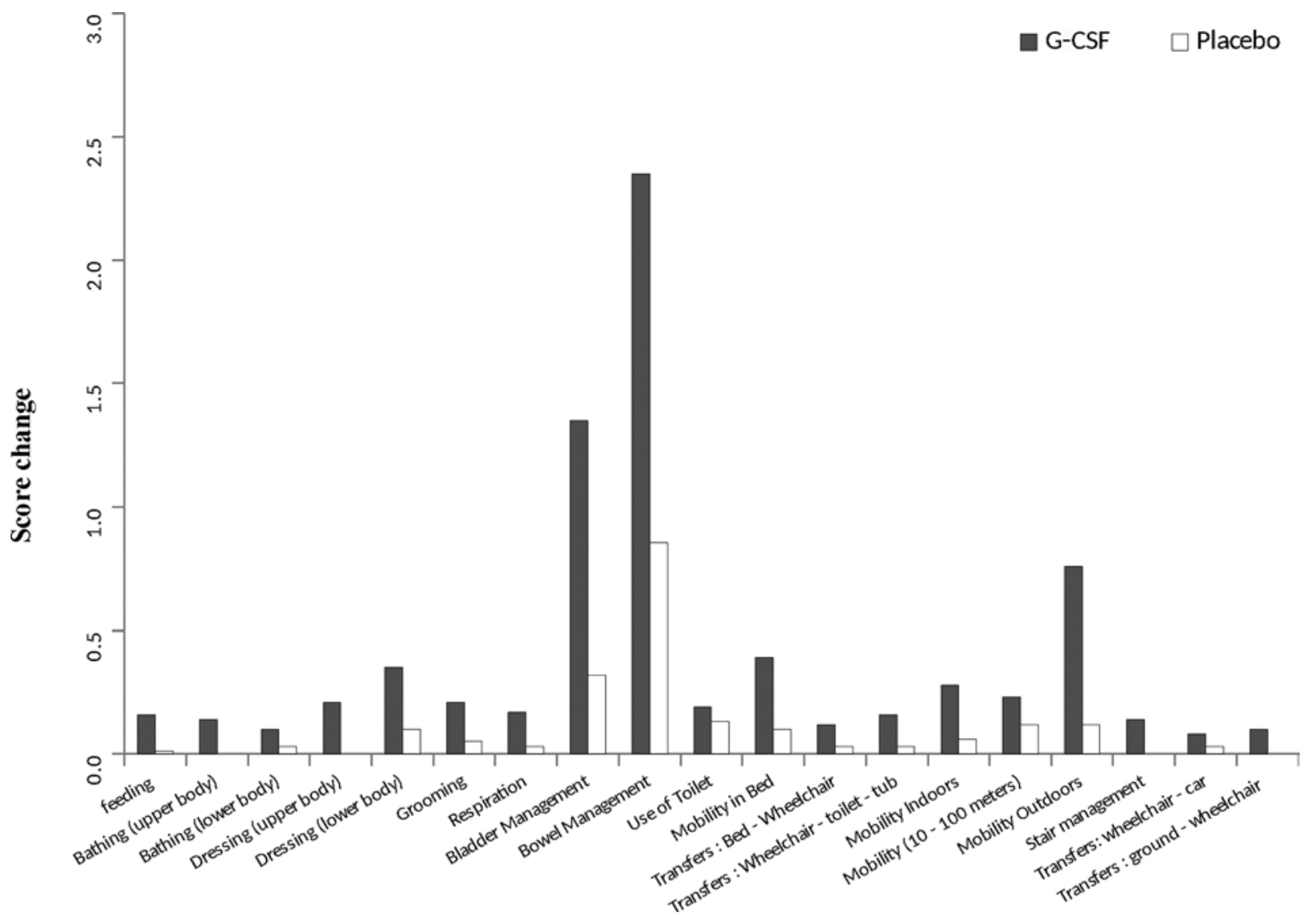

FIG. 3. Spinal cord independence measure (SCIM-III) score changes in the G-CSF and placebo groups. All the changes were statistically significant $(p<0.05)$ except on the Bathing lower body, Respiration, Use of toilet, Bed wheelchair transfers, Wheelchairtoilet-tub transfer, Mobility indoors, Mobility 10-100 m, Wheelchair-car transfers, and Ground-wheelchair transfers subscales. 


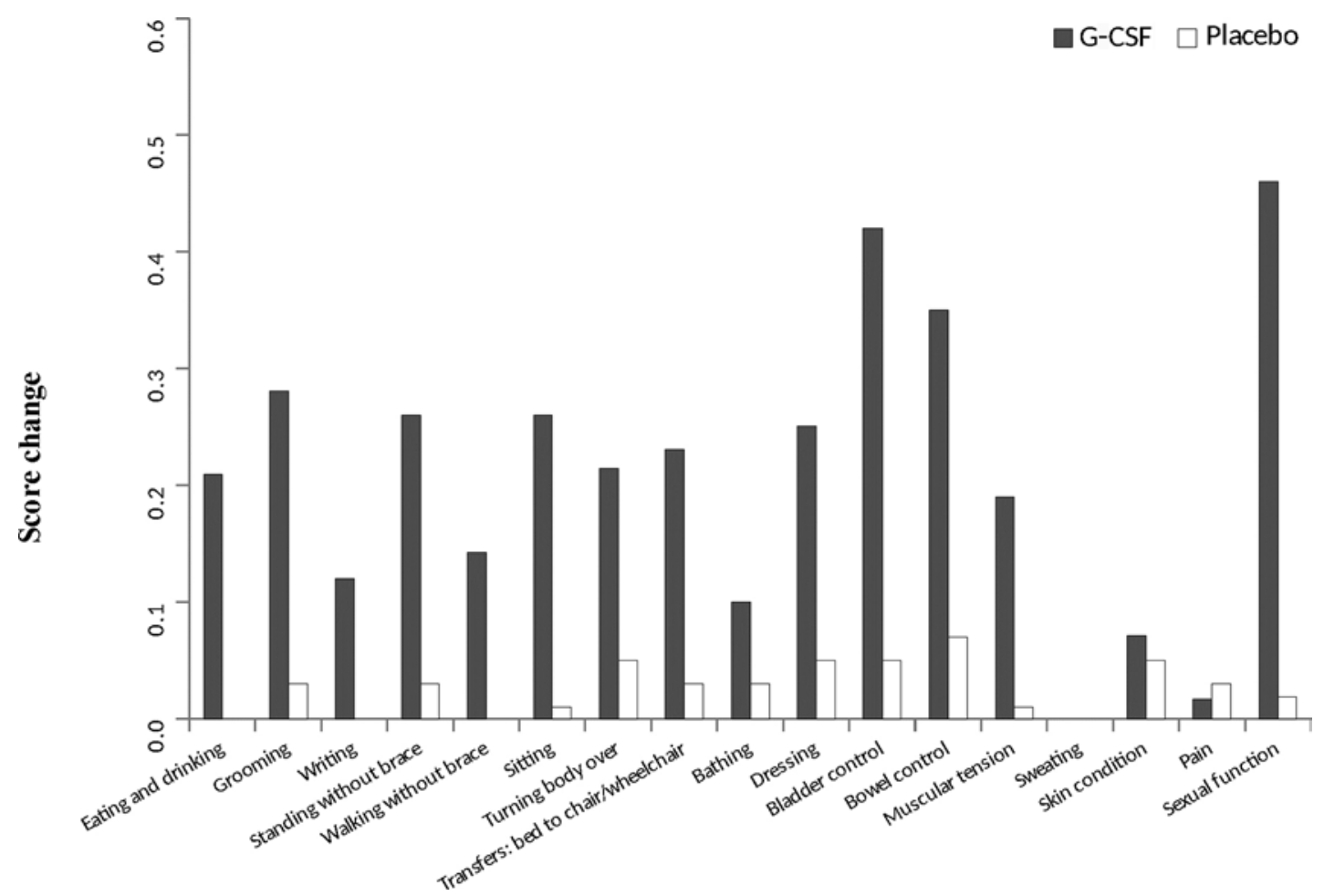

FIG. 4. Functional rating scale (IANR-SCIFRS) score changes in the G-CSF and placebo groups. All the changes were statistically significant $(p<0.05)$ except on the Bathing, Sweating, Skin condition, and Pain subscales.

spasticity 3 months after injection: 3 showed an increase of 1 score level in MAS and 1 showed only increased frequency of spasticity episodes. Also 1 patient (1.7\%) had a decrease in neuropathic pain and another (1.7\%) had decreased spasticity.

\section{Discussion}

Granulocyte-colony stimulating factor (G-CSF), a 19.6$\mathrm{kD}$ glycoprotein, has growth-stimulant properties for activation and differentiation of bone marrow granulocytes, $7,28,38$ and has been previously used in patients with SCI as well as in stroke patients. ${ }^{14,31,37}$ The mechanism of action was speculated to be suppression of neuronal and oligodendroglial apoptosis, thereby protecting myelin sheaths.

G-CSF can also inhibit expression of inflammatory cytokines such as TNF- $\alpha$ and IL-1 $\beta$, and it promotes angiogenesis. ${ }^{23,24,39}$ Chung et al. ${ }^{7}$ found that G-CSF suppressed glial scar formation after SCI in rats, possibly by restricting the expression of glial fibrillary acidic protein and chondroitin sulfate proteoglycans, which might facilitate functional recovery from SCI. Guo et al. ${ }^{17}$ found that G-CSF improves neuronal survival by regulating nucleophosmin-1 (NPM1) expression. The mechanism of G-CSF neuroprotection in these studies may be related in part to attenuation of neuronal apoptosis by NPM1 expression.

Review of the literature shows a credible body of experimental evidence indicating that G-CSF has a robust effect on SCI in different experimental models and species. ${ }^{9}$ Koda et al. ${ }^{26}$ have shown that G-CSF mobilizes bone marrow-derived stem cells into the peripheral blood. It is speculated that homing of these cells in the injured spi- nal cord promotes functional recovery after compressioninduced SCI in mice. Kawabe et al. ${ }^{24}$ showed that G-CSF exerts neuroprotective effects via promotion of angiogenesis after SCI in rat spinal cord. Also 2 other studies ${ }^{12,30}$ have shown that G-CSF improves functional outcome and motor recovery after experimental SCI by counteracting apoptosis and enhancing connectivity in the injured spinal cord. With its demonstrated antiapoptotic activity, G-CSF could help to preserve neuronal tissue around the site of injury. ${ }^{12}$ In addition to the corticospinal tract, serotonergic fibers are thought to be of major importance for the recovery of motor function that has been observed following G-CSF administration in animal models of SCI. ${ }^{30}$

The mechanisms of G-CSF action appear to be both neuroprotective and neuroregenerative. In chronic incomplete SCI, there may be hibernating nonfunctional axons that may be induced to start to function again with G-CSF treatment.

Based on the above results, some clinical trials have been initiated for the treatment of SCI at various phases. As far as we know, 6 clinical trials of G-CSF administration for SCI patients have been conducted. There have been 2 trials - one with phase I/IIa design ${ }^{34}$ and one an openlabel multicenter prospective controlled clinical trial ${ }^{35}-$ of G-CSF for patients with worsening symptoms of cervical and/or thoracic compression myelopathy. There have also been 2 trials - a phase I/IIa trial ${ }^{39}$ and an open-label multicenter prospective nonrandomized controlled clinical trials ${ }^{21}$ - for acute TSCI. Finally, 2 trials-one phase I and one phase II-for chronic TSCI have been conducted., ${ }^{9,32}$ Methodologically, this study is the first randomized con- 
TABLE 3. Subgroup analysis of changes in neurological (based on ASIA) and functional (based on SCIM-III and IANRSCIFRS) scores in the G-CSF and placebo groups according to neurological level (cervical or thoracolumbar)

\begin{tabular}{ccrr}
\hline Variable & $\begin{array}{c}\text { G-CSF Group } \\
(n=56)\end{array}$ & $\begin{array}{r}\text { Placebo Group } \\
(n=58)\end{array}$ & $\begin{array}{c}p \\
\text { Value }\end{array}$ \\
\hline Cervical level $(n=58)^{*}$ & & & \\
\hline Motor & $6.3 \pm 1.0$ & $1.0 \pm 0.35$ & $<0.001$ \\
\hline Light touch & $7.2 \pm 1.8$ & $1.5 \pm 0.92$ & 0.008 \\
\hline Pinprick & $10.9 \pm 2.7$ & $0.90 \pm 0.64$ & 0.001 \\
\hline SCIM III & $7.2 \pm 1.2$ & $1.2 \pm 0.33$ & $<0.001$ \\
\hline IANR-SCIFRS & $3.8 \pm 0.56$ & $0.30 \pm 0.10$ & $<0.001$ \\
\hline $\begin{array}{c}\text { Thoracolumbar level } \\
\text { (n = 56) } \dagger\end{array}$ & & & \\
\hline Motor & $4.7 \pm 0.71$ & $0.46 \pm 0.71$ & $<0.001$ \\
\hline Light touch & $5.0 \pm 1.3$ & $1.0 \pm 0.47$ & 0.007 \\
\hline Pinprick & $6.5 \pm 1.3$ & $0.89 \pm 0.63$ & $<0.001$ \\
\hline SCIM III & $7.8 \pm 1.4$ & $3.1 \pm 0.97$ & 0.009 \\
\hline IANR-SCIFRS & $3.2 \pm 0.51$ & $0.53 \pm 0.23$ & $<0.001$ \\
\hline
\end{tabular}

Data are mean \pm SE

* Includes 28 patients in the G-CSF group and 30 in the placebo group.

$\dagger$ Includes 28 patients in each group.

trolled clinical trial of G-CSF administration in patients with TSCI.

Historically, in many studies, the severity of SCI has been proposed to be one of the most important prognostic factors for neurological recovery after acute phase treatments of SCI. Patients with AIS grade A injuries have 6\%$13 \%$ chance of neurological recovery, while neurological recovery can be expected for approximately two-thirds of patients who have AIS grade B injuries after initial resuscitation, steroids, and decompression. ${ }^{18}$ Consequently, incomplete SCI is prognostically more favorable for restoration of spinal cord motor function. ${ }^{6}$ It is not very farfetched to extrapolate this idea for estimation of cell therapy outcomes. Our phase II study documented that G-CSF administration for incomplete SCIs is associated with significantly greater motor and sensory improvement, and also the more severe the initial AIS grade, the less would be the final sensorimotor recovery. ${ }^{32}$ Accordingly, we chose incomplete (AIS B, C, D) cases for this study.

Many authors have considered the risk of neurological decline after invasive cell therapy modalities (such as intramedullary and/or intrathecal injections) for incomplete SCIs, and the SCI clinical trial guidelines advocate performing safety studies in AIS A cases. ${ }^{40}$ Limiting the invasive neuroprotective therapies to complete SCI patients for safety considerations sounds logical, but the price is likely to be reduced efficacy. With upcoming noninvasive neuroprotective treatments, it may be possible to expand the domain of clinical application of these treatments for incomplete SCI cases, as in this study, which was performed after a pilot safety trial of subcutaneous G-CSF safety. ${ }^{9}$

Outcomes of sensorimotor autorecovery after rehabilitation protocols in the chronic phase have not been significant in comparison with acute cases. ${ }^{13,15}$ Therefore our significant results in chronic patients are not attributable to autorecovery.

In preliminary phase I and II studies, we employed a GCSF dosage of $300 \mu \mathrm{g} / \mathrm{day}$, administered subcutaneously for 7 consecutive days - a modest dose of G-CSF that can be safely administered in TSCI outpatients $\mathrm{s}^{9,32}$-although some authors have advocated a dosage of $10 \mu \mathrm{g} / \mathrm{kg} / \mathrm{day}$ administered intravenously. ${ }^{21,35,39}$

The mean $( \pm \mathrm{SD})$ postinjection WBC count in the GCSF group in our study was $29,240( \pm 7750)$ cells $/ \mathrm{mm}^{3}$. In the study by Takahashi et al., ${ }^{39} 1$ day after the start of G-CSF therapy in acute SCI patients, WBC counts rose to $28,600( \pm 3,200)$ cells $/ \mathrm{mm}^{3}$ in the 5- $\mu$ g group and 26,300 ( \pm $6,300)$ cells $/ \mathrm{mm}^{3}$ in the $10-\mu \mathrm{g}$ group. They reported no significant difference in WBC counts between their 2 groups. Therefore, we preferred the lower dosage to decrease the risk of splenomegaly ${ }^{4}$ and/or inadvertent leukostasis.

Our results show that G-CSF administration improves muscle strength in the upper and lower extremities (as demonstrated by ASIA assessment) 1, 3, and 6 months after intervention. Other clinical studies also support this finding. ${ }^{21,39}$ These findings suggest that G-CSF administration may have a neuroprotective effect on the hibernating descending tracts of the white matter and on the gray matter within the injured spinal segments.

Also, light touch and pinprick sensory changes were significant in the G-CSF group ( $p<0.001$ ), and other studies support this finding. ${ }^{33}$

Regarding functional changes, SCIM-III and IANRSCIFRS scores revealed statistically significant changes in the G-CSF group in comparison with the placebo group at the 3 follow-up points (Table 2).

Subgroup analyses of our findings (Table 3 ) showed no significant difference in neurological and functional changes for cervical and thoracolumbar cases. So both levels may benefit from the treatment.

The most important predictor for functional improvement has been severity of SCI. This means that the AIS changes would determine the changes in functional scores. ${ }^{2}$ According to our results, the greatest AIS changes in the G-CSF group were observed among incomplete patients with AIS C SCIs. On the other hand, AIS grade change was uncommon in AIS D cases, compared with AIS B or C incomplete SCIs.

\section{Side Effects}

Previous reports have described some side effects after G-CSF administration. Mild symptoms include low-back and pelvic pain, fever, chills, headache, rash, nausea, vomiting, and mild hepatic dysfunction. Symptoms were transient and disappeared 2 to 7 days after cessation of the drug. ${ }^{3,9,21}$ In our cases, increased neuropathic pain (12.5\%) and spasticity (1.8\%) were among the persistent but treatable side effects.

We suggest that the dose (300 $\mu \mathrm{g} / \mathrm{day})$, duration (7 consecutive days), and route (subcutaneous injection) of G-CSF administration employed in the present study are generally safe for prescription in chronic incomplete TSCI.

\section{Strengths}

First, the randomized controlled design with parallel 
assignment, concealed allocation, and double blinding of examiners and participants has reduced the risk of systematic bias and therefore constitutes a major strength. Secondly, the G-CSF and placebo groups have been treated at a single institution with the same method of rehabilitation and nursing care. Hence, treatment consistency between the 2 groups has not been compromised. Third, the number of patients enrolled in the study was large enough to obtain sufficient statistical power.

Fourth, the pathophysiology and symptoms of SCI vary depending on the spinal level of injury (cervical or thoracolumbar) as well as the severity of the trauma measured by AIS grade. There was no selection bias in the 2 groups because the distributions of initial AIS grade and neurological level of injury were the same in the G-CSF and placebo groups due to the random block design of the study.

Fifth, regarding cost-effectiveness of the treatment, subcutaneous G-CSF administration does not require hospital admission, especially in chronic postrehabilitation cases, and can be performed on an outpatient basis provided the patient is cooperative and well instructed.

\section{Limitations}

There is a probability of information bias due to error related to the assessment tools, including ASIA, SCIMIII, and IANR-SCIFRS. Because these tools are subjective, and in the absence of sufficient cooperation of the patient, the results may deviate from the actual values. Modern paraclinical measures such as high-power MRI tractography may be required.

Another limitation was loss to follow-up of 5 patients. We tried to minimize the number of patients lost to followup with periodic phone calls for establishing and confirming assessment meetings.

\section{Generalizability}

It should be noted that the methodology of the study may be generalized for other noninvasive neuroprotective treatments, especially in patients with incomplete chronic TSCI.

\section{Conclusions}

Granulocyte-colony stimulating factor administration for incomplete chronic TSCI is associated with significant motor, sensory, and functional improvement. A multicenter study would be the next step for establishment of the hypothesis.

\section{Acknowledgments}

The authors would like to acknowledge the patients for their kind participation in the study.

\section{References}

1. Ackerman P, Morrison SA, McDowell S, Vazquez L: Using the Spinal Cord Independence Measure III to measure functional recovery in a post-acute spinal cord injury program. Spinal Cord 48:380-387, 2010

2. Aidinoff E, Front L, Itzkovich M, Bluvshtein V, Gelernter I, Hart J, et al: Expected spinal cord independence measure, third version, scores for various neurological levels after complete spinal cord lesions. Spinal Cord 49:893-896, 2011
3. Anderlini P, Przepiorka D, Seong D, Miller P, Sundberg J, Lichtiger B, et al: Clinical toxicity and laboratory effects of granulocyte-colony-stimulating factor (filgrastim) mobilization and blood stem cell apheresis from normal donors, and analysis of charges for the procedures. Transfusion 36:590595, 1996

4. Becker PS, Wagle M, Matous S, Swanson RS, Pihan G, Lowry PA, et al: Spontaneous splenic rupture following administration of granulocyte colony-stimulating factor (G-CSF): occurrence in an allogeneic donor of peripheral blood stem cells. Biol Blood Marrow Transplant 3:45-49, 1997

5. Brines M, Grasso G, Fiordaliso F, Sfacteria A, Ghezzi P, Fratelli M, et al: Erythropoietin mediates tissue protection through an erythropoietin and common $\beta$-subunit heteroreceptor. Proc Natl Acad Sci U S A 101:14907-14912, 2004

6. Bryukhovetskiy AS, Bryukhovetskiy IS: Effectiveness of repeated transplantations of hematopoietic stem cells in spinal cord injury. World J Transplant 5:110-128, 2015

7. Chung J, Kim MH, Yoon YJ, Kim KH, Park SR, Choi BH: Effects of granulocyte colony-stimulating factor and granulocyte-macrophage colony-stimulating factor on glial scar formation after spinal cord injury in rats. J Neurosurg Spine 21:966-973, 2014

8. Derakhshanrad N, Saberi H, Tayebi Meybodi K, Taghvaei M, Arjmand B, Aghayan HR, et al: Case report: combination therapy with mesenchymal stem cells and granulocyte-colony stimulating factor in a case of spinal cord injury. Basic Clin Neurosci 6:299-305, 2015

9. Derakhshanrad N, Saberi H, Yekaninejad MS, Eskandari G, Mardani A, Rahdari F, et al: Safety of granulocyte colonystimulating factor (G-CSF) administration for postrehabilitated motor complete spinal cord injury patients: an open-label, phase I study. Cell Transplant 22 (Suppl 1):S139-S146, 2013

10. Derakhshanrad N, Vosoughi F, Yekaninejad MS, Moshayedi P, Saberi H: Functional impact of multidisciplinary outpatient program on patients with chronic complete spinal cord injury. Spinal Cord 53:860-865, 2015

11. Derakhshanrad N, Yekaninejad MS, Vosoughi F, Sadeghi Fazel F, Saberi H: Epidemiological study of traumatic spinal cord injuries: experience from a specialized spine center in Iran. Spinal Cord 54:901-907, 2016

12. Dittgen T, Pitzer C, Plaas C, Kirsch F, Vogt G, Laage R, et al: Granulocyte-colony stimulating factor (G-CSF) improves motor recovery in the rat impactor model for spinal cord injury. PLoS One 7:e29880, 2012

13. Fawcett JW, Curt A, Steeves JD, Coleman WP, Tuszynski MH, Lammertse D, et al: Guidelines for the conduct of clinical trials for spinal cord injury as developed by the ICCP panel: spontaneous recovery after spinal cord injury and statistical power needed for therapeutic clinical trials. Spinal Cord 45:190-205, 2007

14. Gibson CL, Jones NC, Prior MJ, Bath PM, Murphy SP: GCSF suppresses edema formation and reduces interleukin-1 $\beta$ expression after cerebral ischemia in mice. J Neuropathol Exp Neurol 64:763-769, 2005

15. Guest J, Harrop JS, Aarabi B, Grossman RG, Fawcett JW, Fehlings MG, et al: Optimization of the decision-making process for the selection of therapeutics to undergo clinical testing for spinal cord injury in the North American Clinical Trials Network. J Neurosurg Spine 17 (1 Suppl):94-101, 2012

16. Guo X, Bu X, Li Z, Yan Z, Jiang J, Zhou Z: Comparison of autologous bone marrow mononuclear cells transplantation and mobilization by granulocyte colony-stimulating factor in experimental spinal injury. Int J Neurosci 122:723-733, 2012

17. Guo Y, Liu S, Wang P, Zhang H, Wang F, Bing L, et al: Granulocyte colony-stimulating factor improves neuron survival 
in experimental spinal cord injury by regulating nucleophosmin-1 expression. J Neurosci Res 92:751-760, 2014

18. Harrop JS, Hashimoto R, Norvell D, Raich A, Aarabi B, Grossman RG, et al: Evaluation of clinical experience using cell-based therapies in patients with spinal cord injury: a systematic review. J Neurosurg Spine 17 (1 Suppl):230-246, 2012

19. Huang H, Xi H, Chen L, Zhang F, Liu Y: Long-term outcome of olfactory ensheathing cell therapy for patients with complete chronic spinal cord injury. Cell Transplant 21 (Suppl 1):S23-S31, 2012

20. Hyun JK, Kim HW: Clinical and experimental advances in regeneration of spinal cord injury. J Tissue Eng 2010: 650857,2010

21. Inada T, Takahashi H, Yamazaki M, Okawa A, Sakuma T, Kato K, et al: Multicenter prospective nonrandomized controlled clinical trial to prove neurotherapeutic effects of granulocyte colony-stimulating factor for acute spinal cord injury: analyses of follow-up cases after at least 1 year. Spine (Phila Pa 1976) 39:213-219, 2014

22. Itzkovich M, Gelernter I, Biering-Sorensen F, Weeks C, Laramee MT, Craven BC, et al: The Spinal Cord Independence Measure (SCIM) version III: reliability and validity in a multi-center international study. Disabil Rehabil 29:19261933, 2007

23. Kadota R, Koda M, Kawabe J, Hashimoto M, Nishio Y, Mannoji C, et al: Granulocyte colony-stimulating factor (G-CSF) protects oligodendrocyte and promotes hindlimb functional recovery after spinal cord injury in rats. PLoS One 7:e50391, 2012

24. Kawabe J, Koda M, Hashimoto M, Fujiyoshi T, Furuya T, Endo T, et al: Neuroprotective effects of granulocyte colonystimulating factor and relationship to promotion of angiogenesis after spinal cord injury in rats: laboratory investigation. $\mathbf{J}$ Neurosurg Spine 15:414-421, 2011

25. Kirshblum SC, Burns SP, Biering-Sorensen F, Donovan W, Graves DE, Jha A, et al: International standards for neurological classification of spinal cord injury (revised 2011). J Spinal Cord Med 34:535-546, 2011

26. Koda M, Nishio Y, Kamada T, Someya Y, Okawa A, Mori $\mathrm{C}$, et al: Granulocyte colony-stimulating factor (G-CSF) mobilizes bone marrow-derived cells into injured spinal cord and promotes functional recovery after compression-induced spinal cord injury in mice. Brain Res 1149:223-231, 2007

27. Nagata S, Tsuchiya M, Asano S, Kaziro Y, Yamazaki T, Yamamoto $\mathrm{O}$, et al: Molecular cloning and expression of cDNA for human granulocyte colony-stimulating factor. Nature 319:415-418, 1986

28. Nicola NA, Metcalf D, Matsumoto M, Johnson GR: Purification of a factor inducing differentiation in murine myelomonocytic leukemia cells. Identification as granulocyte colony-stimulating factor. J Biol Chem 258:9017-9023, 1983

29. Pannu R, Barbosa E, Singh AK, Singh I: Attenuation of acute inflammatory response by atorvastatin after spinal cord injury in rats. J Neurosci Res 79:340-350, 2005

30. Pitzer C, Klussmann S, Krüger C, Letellier E, Plaas C, Dittgen $\mathrm{T}$, et al: The hematopoietic factor granulocyte-colony stimulating factor improves outcome in experimental spinal cord injury. J Neurochem 113:930-942, 2010

31. Ringelstein EB, Thijs V, Norrving B, Chamorro A, Aichner $\mathrm{F}$, Grond $\mathrm{M}$, et al: Granulocyte colony-stimulating factor in patients with acute ischemic stroke: results of the AX200 for Ischemic Stroke trial. Stroke 44:2681-2687, 2013

32. Saberi H, Derakhshanrad N, Yekaninejad MS: Comparison of neurological and functional outcomes after administration of granulocyte-colony-stimulating factor in motor-complete versus motor-incomplete postrehabilitated, chronic spinal cord injuries: a phase I/II study. Cell Transplant 23 (Suppl 1):S19-S23, 2014

33. Saberi H, Firouzi M, Habibi Z, Moshayedi P, Aghayan HR, Arjmand B, et al: Safety of intramedullary Schwann cell transplantation for postrehabilitation spinal cord injuries: 2-year follow-up of 33 cases. J Neurosurg Spine 15:515525,2011

34. Sakuma T, Yamazaki M, Okawa A, Takahashi H, Kato K, Hashimoto M, et al: Neuroprotective therapy using granulocyte colony-stimulating factor for patients with worsening symptoms of compression myelopathy, Part 1: a phase I and IIa clinical trial. Eur Spine J 21:482-489, 2012

35. Sakuma T, Yamazaki M, Okawa A, Takahashi H, Kato K, Hashimoto M, et al: Neuroprotective therapy using granulocyte colony-stimulating factor for patients with worsening symptoms of thoracic myelopathy: a multicenter prospective controlled trial. Spine (Phila Pa 1976) 37:1475-1478, 2012

36. Salzberg CA, Byrne DW, Cayten CG, van Niewerburgh P, Murphy JG, Viehbeck M: A new pressure ulcer risk assessment scale for individuals with spinal cord injury. Am J Phys Med Rehabil 75:96-104, 1996

37. Schäbitz WR, Kollmar R, Schwaninger M, Juettler E, Bardutzky J, Schölzke MN, et al: Neuroprotective effect of granulocyte colony-stimulating factor after focal cerebral ischemia. Stroke 34:745-751, 2003

38. Schneider A, Kuhn HG, Schäbitz WR: A role for G-CSF (granulocyte-colony stimulating factor) in the central nervous system. Cell Cycle 4:1753-1757, 2005

39. Takahashi H, Yamazaki M, Okawa A, Sakuma T, Kato K, Hashimoto M, et al: Neuroprotective therapy using granulocyte colony-stimulating factor for acute spinal cord injury: a phase I/IIa clinical trial. Eur Spine J 21:2580-2587, 2012

40. Tuszynski MH, Steeves JD, Fawcett JW, Lammertse D, Kalichman M, Rask C, et al: Guidelines for the conduct of clinical trials for spinal cord injury as developed by the ICCP Panel: clinical trial inclusion/exclusion criteria and ethics. Spinal Cord 45:222-231, 2007

\section{Disclosures}

The authors report no conflict of interest concerning the materials or methods used in this study or the findings specified in this paper.

\section{Author Contributions}

Conception and design: Saberi, Derkhshanrad. Analysis and interpretation of data: Yekaninejad. Drafting the article: Saberi, Derkhshanrad. Critically revising the article: Saberi, Sheikhrezaei. Reviewed submitted version of manuscript: all authors. Approved the final version of the manuscript on behalf of all authors: Saberi. Statistical analysis: Yekaninejad. Administrative/ technical/material support: Saberi, Joghataei, Sheikhrezaei. Study supervision: Saberi.

\section{Supplemental Information}

\section{Previous Presentations}

A report of interim results was presented at the IXth annual conference of the International Association of Neurorestoratology in Tianjin, China, August 31-September 3, 2016.

\section{Correspondence}

Hooshang Saberi: Imam Hospital, Brain and Spinal Cord Injury Research Center (BASIR), Tehran, Iran. hgsaberi@yahoo.com. 